\title{
Selecting soybean resistant to the cyst nematode Heterodera glycines using simple sequence repeat (microsatellite) markers
}

\author{
S.M.C.G. Espindola', O.T. Hamawaki ${ }^{1}$, A.P. Oliveira ${ }^{2}$, C.D.L. Hamawaki ${ }^{3}$, \\ R.L. Hamawaki ${ }^{4}$ and L.M. Takahashi ${ }^{1}$ \\ ${ }^{1}$ Instituto de Ciências Agrárias, Universidade Federal de Uberlândia, Uberlândia, \\ MG, Brasil \\ 2Instituto de Genética e Bioquímica, Universidade Federal de Uberlândia, \\ Uberlândia, MG, Brasil \\ ${ }^{3}$ Instituto Master Presidente Antonio Carlos, Araguari, MG, Brasil \\ ${ }^{4}$ Department of Plant, Soil \& Agricultural Systems, \\ Southern Illinois University Carbondale, IL, USA \\ Corresponding author: L.M. Takahashi \\ E-mail: I_m_takahashi@hotmail.com \\ Genet. Mol. Res. 15 (1): gmr.15016850 \\ Received May 22, 2015 \\ Accepted November 5, 2015 \\ Published March 11, 2016 \\ DOI http://dx.doi.org/10.4238/gmr.15016850
}

\begin{abstract}
The soybean cyst nematode (SCN) is a major cause of soybean yield reduction. The objective of this study was to evaluate the efficiency of marker-assisted selection to identify genotypes resistant to SCN race 3 infection, using Sat_168 and Sat-141 resistance quantitative trait loci. The experiment was carried out under greenhouse conditions, using soybean populations originated from crosses between susceptible and resistant parent stock: CD-201 (susceptible) and Foster IAC (resistant), Conquista (susceptible) and S83-30 (resistant), La-Suprema (susceptible) and S57-11 (resistant), and Parecis (susceptible) and S65-50 (resistant). Plants were inoculated with $\mathrm{SCN}$ and evaluated according to the female index $(\mathrm{FI})$, those with $\mathrm{FI}<10 \%$ were classified as resistant to nematode infection. Plants were genotyped for SCN resistance using microsatellite
\end{abstract}


markers Sat-141 and Sat_168. Marker selection efficiency was analyzed by a contingency table, taking into account genotypic versus phenotypic evaluations for each line. These markers were shown to be useful tool for selection of SCN race 3 .

Key words: Microsatellite; Single sequence repeats; Soybean; Cyst nematode

\section{INTRODUCTION}

Owing to its high economic value, soybean production has expanded rapidly throughout Brazil; this has been through breeding plants that are better adapted to country's conditions. Generating resistant plant is one of the most efficient and economical methods for controlling the soybean cyst nematode (SCN), one of the main causes of soybean yield reduction (Matsuo et al., 2012). However because of the great genetic variability of this nematode species, the continual use of the same resistant plant variety exerts selection pressure for SCN breeds that are capable of parasitizing the said variety; in order to avoid this, it is necessary to increase genetic diversity of resistant soybean plants (Silva et al., 2007). In Brazil, there is a greater variability of SCN breeds than that found in the United States; around 11 breeds have been identified in Brazil, carrying a high number of virulence genes (Dias et al., 2009).

Biotechnological evolution is of great importance in the development of plant lines resistant to pathogens and pests, and in the generation of commercially available, economically important cultivars while preventing serious phytosanitary problems that may compromise yields. Increased selection efficiency, better knowledge and characterization of the germoplasm, and maximization of genetic gains have been the objectives of plant breeders worldwide; new ways of achieving these objectives are being constantly sought out.

While proceeding with the soybean breeding, lines (plants descendant of only one plant in more than $95 \%$ of homozygosis) were selected and used in this experiment.

Molecular markers are an efficient method for genotypic evaluation of resistance to SCN resistance in plants, permitting selection of polygenic resistance to this pathogen; and are also an important tool to aid the phenotypic selection method (Santana et al., 2014). They can be useful for indirect selection of traits that are difficult to evaluate and/or are highly affected by the environment (Schuster et al., 2001) even in premature segregating generations, allowing earlier selection and thereby reducing wasted time and investment (Borém and Caixeta, 2006). Another consideration for breeding planting material is identifying, locating, and characterizing quantitative trait loci (QTL) associated with agronomically important traits (Concibido et al., 2004).

Microsatellites are useful tools as they facilitate the detection of a large amount of variability (Vieira et al., 2009); are not affected by environmental influence, and are transmitted.

The aim of this study was to evaluate the efficiency of the microsatellite markers Sat_168 and Sat-141 in determine QTL for resistance to the soybean plants to infection with SCN race 3.

\section{MATERIAL AND METHODS}

The experiment was conducted under greenhouse conditions in the experimental station of Syngenta Seeds Ltd., between March 15, 2003 and April 28, 2003. Plant populations were obtained by crossing SCN race 3 resistant and susceptible stock as follows: population 9024 (43 $\mathrm{F}_{5: 6}$ 
lines) was obtained from CD-201 (susceptible) and Foster IAC (resistant); 7103 (38 $\mathrm{F}_{5: 6}$ lines), BR/ MG 46 Conquista (susceptible) crossed with S83-30 (resistant); 7105 (60 lines), from La-suprema (susceptible) crossed with S57-11 (resistant); and 7109 (44 $\mathrm{F}_{5: 6}$ lines) from Parceis (susceptible) crossed with S65-50 (resistant). The source of resistance for lines S57-11 and S65-50 was derived from PI 88788, and line S83-30 and cultivar Foster-IAC, resistance was derived from the cultivar 'Peking'.

Populations were previously subjected to selection aided by molecular markers, in accordance with the internal procedures of Syngenta Seeds Ltd. (commercial in confidence). Microsatellite markers Sat-141 and 163 were used to identify resistance alleles in order to classify the lines as resistant or susceptible. Experiments were designed as complete randomized blocks with six single-plant replicates. For all experiments, seeds of the progenitor lines and varieties were sown into tubettes containing a substrate of soil and sand (1:2). Tubettes were held in 6-L plastic vases (5 per vase) and moistened daily to control temperature.

The SCN inoculum used was obtained from natural soil infested with Heterodera glycines race 3, collected from Lhoman Farm in Irai de Minas, MG. To ensure that eggs were obtained, soil mixed with roots was collected. Roots were separated, placed above a sieve with $0.85-\mathrm{mm}$ size mesh (20 mesh), attached above another sieve with $0.15 \mathrm{~mm}$ (100 mesh), and washed under a water jet. Females retained in the sieve were collected by flushing with water jets from a wash bottle and placed on a sieve of 100 mesh that was used exclusively for crushing cysts and the female nematodes. In order to release the eggs, females were crushed using the bottom of a test tube; water was added so that the eggs passed to a $0.025-\mathrm{mm}$ size mesh (500 mesh) attached below the crushing sieve. Eggs retained in the 500-mesh sieve were collected using jets of sucrose solution ( $454 \mathrm{~g} / \mathrm{L}$ water). The solution containing eggs was then centrifuged at $2400 \mathrm{rpm}$ for $1 \mathrm{~min}$ in a table centrifuge (Excelsa Baby II model 206-R, Fanem, São Paulo, Brazil) and supernatant strained and washed was poured into a sieve of 500 mesh and washed with filter water, to remove the excess sacarose. The eggs were collected into a Becker cup (Uniglas, Uberlândia, Brazil) and the suspension was calibrated with the aid of the Peters layer (Astel, Botucatu, Brazil), to contain 359 eggs $/ \mathrm{mL}$.

The inoculation was performed 10 days after the seeding, when the soybean seedling reached stage VI. Three holes were with a depth of $1.5 \mathrm{~cm}$ and a diameter of $0.5 \mathrm{~cm}$ was made on the pot containing plants, equidistant from each other. Next $2 \mathrm{~mL}$ of the egg suspension was placed in each hole with the aid of a syringe without a needle.

The number of female nematodes present in the roots of each plant was determined 33 days after inoculation. Plants were carefully removed from tubettes and the root systems washed beneath a water jet on a 20-mesh sieve above a 100-mesh sieve. Females retained on the $100-$ mesh sieve were collected, with the aid of water jets, into a simple glass cup. A soil sample (200 $\mathrm{cm}^{3}$ ) was also collected from each tubette and added to a container with $1 \mathrm{~L}$ water. Soil harrowed to promote mixing with water to generate a suspension, allowed to rest for $15 \mathrm{~s}$, and then poured onto the 20-mesh sieve above a 100-mesh sieve and cysts retained on the 100-mesh sieve were collected into a cup using water jets. Suspensions from root and soil samples were filtered through demarcated filter paper, and examined using a stereoscopic microscope to count the number of females (from root samples) and cysts (soil samples). Reaction of the plant lines were determined based on the index of females (FI), proposed by Golden et al. (1970), for each line, roots were collected and the estimated number of females was counted, if less than $10 \%$, the line was considered resistant, to be considered susceptible, this number should be more than $10 \%$.

This FI was obtained by calculating the number of females found in the line divided by the number of females found in the susceptible parental multiplied by 100 . Plant lines were ranked according to reaction to the cyst nematode beginning with the evaluation of the females (FI), which 
was lower than $10 \%$. The broad sense heritabilities were evaluated for selection based on the averages of the treatments (lineages of soybean), utilizing the estimates of the variance components, using the program SAS (SAS Institute Inc., 2002, USA), calculating the variance components among the progenies.

Significance of the deviations was verified by the chi-squared test, using the following expression:

$$
x^{2}=\frac{\sum(\mathrm{No}-\mathrm{Ne})^{2}}{\mathrm{Ne}}
$$

In the formula above, No is the number observed, and Ne the number expected.

For statistical analysis, data were subjected to the transformation to $[\sqrt{ }(x+1 / 2)]$, where $x$ is the number of nematode females/plant tested by analysis of variance using the $F$ test. Averages were clustered using the Scott Knott test at a probability level of $5 \%$.

\section{RESULTS AND DISCUSSION}

Breeding for resistance to the cyst nematode brings a few complications, when based only on the phenotypic analysis of the trait in question. Plant selected in initial generations, through phenotypic analysis alone, would probably segregate in following generations due to the heterozygous site. Thus, the selection based on both phenotype and genotype (selection assisted by molecular markers), is required to ensure stable resistance. All of the populations behaved in a different way with regards to the ranking of the resistant $(\mathrm{FI}<10 \%)$ and susceptible $(\mathrm{FI}>10 \%)$ lines. In population 9024, only two lines presented reaction of resistance to NCS race 3.

To aid in the interpretation of this data, some parameters were evaluated, such as sensitivity, specificity, the positive predicted value, the negative predicted value, and the efficiency or precision of the markers (Table 1). Population 7105 presented greater variation in the average number of females and a greater number of resistant genotypes. In population 71096 that are lines resistant to NCS were observed.

Table 1. Parameters for assessing performance of Sat-141 and 168 in populations $9024,7103,7105$, and 7109.

\begin{tabular}{l|l}
\hline Terms & Description \\
\hline Positive diagnosis & Resistance alleles present \\
\hline Negative diagnosis & Resistance alleles absent \\
\hline Sensitivity & Proportion of correct positives (true positive) of the total positive cases \\
\hline Specificity & Proportion of correct diagnoses negative (true negatives) totaling negative cases \\
\hline Positive predictive value & Proportion of positives correct of total diagnoses (phenotypic) positive \\
\hline Negative predictive value & Proportion of negative diagnoses correct of total diagnoses (phenotypic) negative \\
\hline Precision or accuracy & Proportion of correct diagnoses (positive and negative) of the total diagnostics \\
\hline
\end{tabular}

Estimate of heritability is a very useful parameter for breeding programs. It permits prediction of the likely success of selection, reflecting the proportion of phenotypic variation as an indicator of the reproductive factor. This parameter was estimated for populations 9024, 7103, 7105 , and 7109 as respectively $46,28,66$, and $77 \%$ heritability for resistance to SCN race 3 (Table 2 ). These values indicate the proportion of the variation of the number of females, which is of a genetic nature; thus, one can observe that populations 7105 and 7109 suffered less influence from environmental factors, compared with the other two populations. 
Table 2. Average number of females (ANF); female index (FI\%); parent reactions and soybean lines of the populations 9024, 7103, 7105 and 7109; average number of amplitude index of females and females and heritability $\left(h^{2}\right)$; Uberlândia, 2015.

\begin{tabular}{l|c|c|c|c|c|c|c}
\hline \multirow{2}{*}{ Population } & \multirow{2}{*}{ ANF } & \multirow{2}{*}{$\mathrm{FI}$} & \multicolumn{2}{|c|}{ Number of lines } & \multicolumn{2}{|c|}{ Amplitude } & \multirow{2}{*}{$h^{2}$} \\
\cline { 4 - 7 } & & & Resistant (R) & Susceptible (S) & ANF (R) & FI (S) & \\
\hline 9024 & $0-25.17$ & $0-290.4$ & 3 & 41 & $0-0.5$ & $19.2-290.4$ & 46 \\
\hline 7103 & $0.5-26.67$ & $4.5-242.4$ & 0 & 38 & 0 & $11.4-242.4$ & 28 \\
\hline 7105 & $0-16.75$ & $0-72.8$ & 25 & 35 & $0-2.2$ & $10.1-72.8$ & 66 \\
\hline 7109 & $0.2-24.5$ & $2-250$ & 6 & 38 & $0.2-0.8$ & $13.6-227.9$ & 77 \\
\hline
\end{tabular}

Populations were analyzed phenotypically, and then genotyped for resistance to SCN race 3, using microsatellite markers Sat_168 and Sat-141, this latter is in the group of molecular connection $G$ of the soybean developed by Cregan et al. (1999), located at a distance of $1.5 \mathrm{cM}$ from the group of the main soybean nematode resistance site, locus Rhg 1. The marker Sat-168 is situated at $0.4 \mathrm{cM}$ from Rhg1 locus and in connection B. The efficiency or precision of these markers in distinguishing $\mathrm{SCN}$ race 3-resistant lines was analyzed in the four study plant populations by a contingency table of genotype analyzed by microsatellites, versus phenotype analysis.

For population 9024, the microsatellite markers presented, jointly, a precision of $45 \%$ (17/40) (Table 3). On the other hand, it showed high specificity of $100 \%$ (17/17) (Table 3) and did not present a false negative. Thusly, one can affirm that in population 9024 the microsatellites Sat_168 and Sat-141 presented a probability of $100 \%$ of not detecting the site of resistance to SCN race 3 , when it is not present. In this population by presenting high specificity and a low sensitivity of $4.3 \%(1 / 23)$ and yet did not present a false-negative result. These markers are more efficient in diagnosing the absence of the resistance site. While the probability that the markers will be connected to the resistance site is small (4.35\%), when this occurs, the probability that there will be an erroneous diagnosis is null as its positive predictive value is $100 \%(1 / 1)$ (Table 4).

Table 3. Contingency table comparing phenotypic analysis for resistance to $\mathrm{SCN}$ race 3 , and genotypic analysis with Sat-141 and 168, for populations 9024, 7103, 7109, and 7105; Uberlandia, 2003.

\begin{tabular}{|c|c|c|c|}
\hline \multirow{2}{*}{ Greenhouse } & \multicolumn{2}{|c|}{ Sat-141 and 168} & \multirow[b]{2}{*}{ Total } \\
\hline & Resistance gene absent & Resistance gene present & \\
\hline $\begin{array}{l}\text { Population } 9024 \\
\text { Susceptible }\end{array}$ & (a) & (1) & 39 \\
\hline Resistant & 00 & 01 & 01 \\
\hline Total & 17 & 23 & 40 \\
\hline$\chi^{2}=0.02$ & $\mathrm{P}<0,05$ & Model: 9:7 & \\
\hline \multicolumn{4}{|l|}{ Population 7103} \\
\hline Susceptible & 10 & 27 & 37 \\
\hline Resistant & 00 & 00 & 00 \\
\hline Total & 10 & 27 & 37 \\
\hline$\chi^{2}=1.29$ & $\mathrm{P}<0,05$ & Model: 3:1 & \\
\hline \multicolumn{4}{|l|}{ Population 7109} \\
\hline Susceptible & 11 & 24 & 35 \\
\hline Resistant & 02 & 03 & 05 \\
\hline Total & 13 & 27 & 40 \\
\hline$\chi^{2}=0.14$ & $\mathrm{P}<0,05$ & Model: 9:7 & \\
\hline \multicolumn{4}{|l|}{ Population 7105} \\
\hline Susceptible & 31 & 04 & 35 \\
\hline Resistant & 11 & 13 & 24 \\
\hline Total & 42 & 17 & 59 \\
\hline$\chi^{2}=0.35$ & $\mathrm{P}<0,05$ & Model: 3:1 & \\
\hline
\end{tabular}


For population 9024 using the chi-squared test, the hypothesis that markers segregate in accordance with the double-recessive epistasis model (9:7) (Table 3), through recombination between two independent genes with two alleles each, was accepted. Thus, resistance only occurred when there was joint participation at the two sites. In the case of susceptibility, individuals possess a complete absence of the dominant alleles or only one dominant allele at one of the sites.

For population 7103 , it was accepted the hypothesis of segregation $3: 1$ by the test of $\chi^{2}$ (Table 3). This proportion was characteristic of the complete dominance model between the resistant allelles. In this case, the dominant homozygote and heterozygote presented the same resistant phenotype. Susceptible individuals possessed the recessive homozygous genotype. Population 7105 also fitted the complete dominance model (Table 3); however, unlike 7103, the trait of resistant was recessive.

For population 7109 (Table 3), the complete dominance (3:1) hypothesis was accepted as well as the double-recessive epistasis hypothesis (9:7), with dominance for the resistance trait.

For population 7103, markers Sat-141 and 168 presented precision of $27.03 \%(10+0 / 37$; Table 3). While a high specificity of $100 \%$ was observed, the sensitivity was null $(0 / 27)$. In that population, therefore, the probability that the markers correctly identified the lack of the site of resistance was 100\%; in other words, false-negative diagnoses did not occur. However, the probability that the opposite will occur is $0 \%$, which is the sensitivity of the markers.

Table 4. Summary of data collected with markers Sat_168 and Sat-141 for populations $9024,7103,7105$, and 7109; Uberlandia, 2003.

\begin{tabular}{l|c|c|c|c}
\hline \multirow{2}{*}{ Parameters } & \multicolumn{4}{|c}{ Populations } \\
\cline { 2 - 5 } & 9024 & 7103 & 7105 & 7109 \\
\hline Sensitivity (\%) & 4,3 & 0,0 & 73,8 & 11,1 \\
\hline Specificity (\%) & 100,0 & 100,0 & 54,1 & 84,6 \\
\hline Positive predictive value (\%) & 100,0 & 0,0 & 88,5 & 60,0 \\
\hline Negative predictive value (\%) & 43,5 & 27,0 & 74,5 & 32,0 \\
\hline Precision or accuracy (\%) & 45,0 & 27,0 & 74,0 \\
\hline
\end{tabular}

The predictive values indicate that the markers are in fact making a correct diagnosis, that is to say, if positive, it indicates the presence of the resistant site; if negative, it indicates the absence. A high predictive value means high probability of success and vice versa (Vieira, 2003). The predictive value of the DNA (deoxyribonucleic acid) marker depends on the connection with the resistance gene and the phenotypic expression of the gene in other genetic background and environments, and need to be tested in more populations (Prabhu et al., 1999).

Contrary to population 9024, population 7103 presented low positive and negative predictive values of 0 and $27 \%$, respectively (Table 4). This indicates a high rate of false-positive diagnosis, which does not inspire confidence in the diagnosis made by these markers in this population.

One of the principal inconveniences in the selection aided by molecular markers happens when the marker used to select is distant from the gene of interest, increasing the "crossing-over" (exchange of genetic material between chromatides) between the marker and the gene. This results in a high percentage of false-positive and/or false-negative diagnoses (Mohan et al., 1997).

The markers Sat-141 and Sat-168 present a precision of $74.58 \%(31+13 / 59)$ in distinguishing the phenotypes that are resistant and/or susceptible to SCN race 3 in population 7105 (Table 4). This same population still presented a high negative predicted value of $88.75 \%(31 / 35)$. Thus one can say that the probability of having a correct positive diagnosis (positive predictive value $=54.17 \%$ ) is less than the probability of having a correct negative diagnosis $(88.57 \%)$. High rates of sensibility and specificity were observed, 76.47 and $73.81 \%$ respectively. This reflected in 
a smaller number of false-positive diagnoses. Presenting a precision of 74.58 and yet a negative predictive value of $88.57 \%$, the microsatellites used present good performance in this population. In this case, the chance of successfully detecting the absence of resistance is greater $(88.57 \%)$ than the chance of successfully selecting resistant plants (54.17\%) (Table 4).

For population 7109 , the microsatellites Sat-141 and Sat-168 presented a precision of only $35 \%$ (14/40) (Table 4). Furthermore, the sensitivity was $11.11 \%(3 / 27)$, which reflects in a high index of false-positive results (24). On the other hand, a specificity of $84.61 \%(11 / 13)$ was obtained, in other words, it does not indicate the presence of the resistance allele when it is not present. This high specificity brings, as a consequence, the low occurrence of false-negative diagnoses. The positive predictive value (3/5) indicates that there was $60 \%(3 / 5)$ of probability of success in the positive diagnoses.

\section{Conflicts of interest}

The authors declare no conflict of interest.

\section{ACKNOWLEDGMENTS}

The authors thank Syngenta Seeds Ltd. for providing scientific technical support, and CNPq, which financed this study.

\section{REFERENCES}

Borém A and Caixeta E (2006). Marcadores moleculares. Editora Jard. 1: 307-374.

Concibido VC, Diers BW and Arelli PR (2004). A decade of QTL mapping for cyst nematode resistance in soybean. Crop Sci. 44: 1121-1131. http://dx.doi.org/10.2135/cropsci2004.1121

Cregan PB, Mudge J, Fickus EW, Daneh D, et al. (1999). Two simple sequence repeat markers to select for soybean cyst nematode resistance conditioned by the rhg1. Theor. Appl. Genet. 99: 811-818. http://dx.doi.org/10.1007/s001220051300

Dias WP, Silva JEV, Carneiro ES, Garcia A, et al. (2009). Nematóide de cisto da soja: biologia e manejo pelo uso da resistência genética. Nematol. Bras. 33: 1-16.

Golden AM, Epps JM, Riggs RD, Duclo LA, et al. (1970). Terminology and identity of intraspecific forms of the soybean cyst nematode (Heterodera glycines). Plant Dis. Rep 54: 544-546.

Matsuo E, Sediyama T, Oliveira RDL, Cruz CD, et al. (2012). Avaliação de genótipos de soja em relação ao nematóide do cisto. Bragantia 71: 173-181. http://dx.doi.org/10.1590/S0006-87052012005000016

Mohan M, Nair RS, Bhagwat A, Krishna TG, et al. (1997). Genome mapping, molecular markers and marker-assisted selection in crop plants. Mol. Breed. 3: 87-103. http://dx.doi.org/10.1023/A:1009651919792

Prabhu RR, Njiti VN, Bell-Johson B, Johnson JE, et al. (1999). Selecting soybeans cultivars for dual resistance to soybean cyst nematode and sudden death syndrome using two DNA markers. Crop Sci. 39: 982-987. http://dx.doi.org/10.2135/crops ci1999.0011183X003900040005x

Santana FA, Silva MF, Guimarães JKF, Ferreira MFS, et al. (2014). Marker-assisted selection strategies for developing resistant soybean plants to cyst nematode. Crop Breed. Appl. Biotechnol. 14: 180-186. http://dx.doi.org/10.1590/1984$\underline{70332014 \mathrm{v} 14 \mathrm{n} 3 \mathrm{a} 27}$

Schuster I, Abdelnoor RV, Arim SRR, Carvalho VP, et al. (2001). Identification of a new major QTL associated with resistance to soybean cyst nematode (Heterodera glycines). Theor. Appl. Genet. 102: 91-96. http://dx.doi.org/10.1007/s001220051622

Silva MF, Schuster I, Cervigni GDL, Silva JFV, et al. (2007). Inheritance of resistance to soybean cyst nematode races 3 and 14 in soybean RIL and F2 populations. Pesq. Agropec. Bras. 42: 1735-1740. http://dx.doi.org/10.1590/S0100$\underline{204 X 2007001200010}$

Vieira ESN, Schuster I, Silva RB and de Oliveira MAR (2009). Genetic variability of soybean cultivars obtained through microsatellite markers in agarose gel. Pesq. Agropec. Bras. 44: 1460-1466. http://dx.doi.org/10.1590/S0100$\underline{204 \times 2009001100013}$

Vieira S (2003). Bioestatística: tópicos avançados. Campos/Elsevier, 78-81. 